\title{
Testing Euclidean Spanners
}

\author{
Frank Hellweg Melanie Schmidt Christian Sohler \\ Department of Computer Science \\ Technical University of Dortmund \\ 44227 Dortmund, Germany
}

\begin{abstract}
In this paper we develop a property testing algorithm for the problem of testing whether a directed geometric graph with bounded (out)degree is a $(1+\delta)$-spanner.
\end{abstract}

\section{Introduction}

Property testing is the computational task of deciding whether a given object has a predetermined property $\Pi$ or is far away from every object with property $\Pi$. Thus, property testing can be viewed as a relaxation of a standard decision problem. The main goal of property testing is to develop randomized algorithms that perform this relaxed decision task by only looking at a small part of the input object, i.e. we want to develop algorithms whose running time is sublinear in the object's description size.

Property testing has been introduced by Rubinfeld and Sudan [30] and the study of combinatorial properties has been initiated by Goldreich, Goldwasser, and Ron [23]. Since then, property testing algorithms have been developed for properties of functions [22, 21,11 , properties of distributions [8,7], algebraic properties [26], graph and hypergraph properties $[23,3,14,10]$, and geometric properties. In this paper we continue the study of property testing algorithms for geometric properties. Previous work on geometric property testing includes testing algorithms for convexity of polygons [18], convexity [29], geometric properties of point sets (for example convex position) and the Euclidean minimum spanning tree $[16,15,17]$, and clusterability of point sets $[1,15]$.

Our contribution In this work, we develop property testing algorithms for Euclidean spanners. A weighted directed geometric graph $(P, E)$ is a directed graph whose vertex set is a set of points in the Euclidean space $\mathbb{R}^{d}$ and whose edge weights (lengths) are given by the Euclidean distance of the vertices, i.e. edge $[p, q\rangle$ has length $\|p-q\|_{2}$. A graph is called $(1+\delta)$-spanner, if for every pair of vertices $p, q$ the shortest path distance $d_{G}(p, q)$ in $G$ is at most $(1+\delta) \cdot\|p-q\|_{2}$, i.e. the shortest path distance in $G$ is a good approximation of the true distance of the points $p$ and $q$. Euclidean spanners are a fundamental geometric 
graph structure as they can be used to approximately solve many geometric proximity problems, and they find applications, for example, in the area of mobile ad-hoc networks. Many different constructions of Euclidean spanners are known. Euclidian spanners with a linear number of edges can for example be constructed by using so-called $\Theta$-graphs [13, 25] or structures based on the well-separated pair decomposition [12, 28]. Also techniques to construct spanners with bounded-degree are known [5]. For more details we refer to the book [28]. We investigate the question whether a given graph is a Euclidean spanner. The related question of computing the stretch factor of a given graph has recently been studied in [4, 19, 27]. Additionally, Ahn et al. [2] discuss the problem to find an edge whose removal leads to the smallest possible increase in the stretch factor, and Farshi et al. [20] consider the question which edge should be added to receive the best decrease in the stretch factor (both articles consider very special cases only).

We say that a geometric graph $G$ is $\epsilon$-far from a $(1+\delta)$-spanner, if one has to insert more than $\epsilon n$ edges into $G$ to make it a $(1+\delta)$-spanner. A property tester is a randomized algorithm that has to distinguish $(1+\delta)$-spanners from graphs which are $\epsilon$-far from any $(1+\delta)$-spanner using a sublinear number of queries to the graph, which is assumed to be stored in adjacency list representation.

In this paper, we show that the property of being a $(1+\delta)$-spanner can be tested with $\tilde{\mathcal{O}}\left(\delta^{-3 d} \epsilon^{-3} \sqrt{n}\right)$ queries for bounded (out)degree graphs.

\section{Preliminaries}

Let $G=(P, E)$ be a directed geometric graph with vertex set $P:=\{1, \ldots, n\}$ and edge set $E$, where the vertices of $P$ are points in the $\mathbb{R}^{d}$ and $d \in \mathbb{N}$ is a constant. We use $[p, q\rangle$ to denote a directed edge from $p$ to $q$. The length of an edge $[p, q\rangle$ is defined to be the Euclidean distance $\|p-q\|_{2}$ between $p, q$, i. e. the edge lengths are induced by the positions of vertices. We use $d_{G}(p, q)$ to denote the shortest path distance from $p$ to $q$, i.e. $d_{G}(p, p)=0$, $d_{G}(p, q)=\|p-q\|_{2}$, if $[p, q\rangle \in E$ and $d_{G}(p, q):=\min _{\text {paths } Q \text { from } p \text { to } q} \sum_{\left[p^{\prime}, q^{\prime}\right\rangle \in Q} d_{G}\left(p^{\prime}, q^{\prime}\right)$ else. We assume that $G$ has an outdegree of at most $D \in \mathbb{N}$ and that it is stored in the adjacency list model [24], i. e. we have access to a function $f_{G}: P \times\{1, \ldots, D\} \rightarrow P \cup\{+\}$, where $f_{G}(p, i)$ returns the $i$-th neighbor of vertex $p$ if $p$ has at least $i$ neighbors and + otherwise.

Definition 1 Let $\delta>0$ be a parameter. A geometric graph $G$ is called a $(1+\delta)$-spanner, if $d_{G}(p, q) \leq(1+\delta)\|q-p\|_{2}$ for all pairs of vertices $[p, q\rangle \in P^{2}, p \neq q$.

In this paper we will assume that $0<\delta<1$.

Definition 2 Let $G$ be a directed geometric graph and let $0<\epsilon<1$. A graph $G$ is $\epsilon$-far from being a $(1+\delta)$-spanner, if one has to insert more than $\in$ n edges to make $G a(1+\delta)$ spanner (note that there is no restriction to maintain the degree bound D). G is $\epsilon$-close to being a $(1+\delta)$-spanner, if it is not $\epsilon$-far from it.

An algorithm $\mathcal{A}$ is called a property tester with one-sided error for the property of being a $(1+\delta)$-spanner, if for any directed geometric graph $G$ it outputs 
- true with a probability of 1 , if $G$ is a $(1+\delta)$-spanner

- false with probability at least $2 / 3$, if $G$ is $\epsilon$-far from being a $(1+\delta)$-spanner

when it is given $n, \delta$ and $\epsilon$ as input and oracle access to $f_{G}$. The query complexity of $\mathcal{A}$ is the worst-case number of accesses to $f_{G}$ it needs.

\section{The algorithm}

Our algorithm for testing geometric spanners works as follows. We first sample a set of $s=\tilde{\mathcal{O}}\left(\frac{\sqrt{n}}{\delta \mathcal{O}(1) \epsilon^{\mathcal{O}(1)}}\right)$ vertices $p_{1}, \ldots, p_{s}$ uniformly at random. Then we start a shortest path computation from each vertex using Dijkstra's algorithm until $O\left(\log n /\left(\epsilon \delta^{2 d}\right)\right)$ vertices have been visited. We call this traversal Dijkstra traversal. Finally, we check for every sample point $p_{i}$ if there exists another sample point $p_{j}$ within a distance of $W /(1+\delta)$ such that $(1+\delta) \cdot\left\|p_{i}-p_{j}\right\|_{2}>d_{G^{\prime}}\left(p_{i}, p_{j}\right)$, where $G^{\prime}$ is the graph induced by the Dijkstra traversals, i.e. $G^{\prime}$ contains exactly the vertices and edges that have been visited during all such traversals, and $W$ is the maximal graph distance reached during the traversal.

\section{UNIFORMTESTER $(n, G, \delta, \epsilon)$}

Sample $s=\tilde{\mathcal{O}}\left(\delta^{-d} \epsilon^{-2} \sqrt{n}\right)$ points $p_{1}, \ldots, p_{s}$ from $P$ u.i.d. without replacement for $i \leftarrow 1$ to $s$

Perform a Dijkstra traversal in $G$ from $p_{i}$ until $s^{\prime}=\tilde{\mathcal{O}}\left(\delta^{-2 d} \epsilon^{-1}\right)$ nodes have been visited

Let $R$ be the set of vertices visited and let $W=\max _{q \in R} d_{G}\left(p_{i}, q\right)$

forall points $p_{j}$ such that $\left\|p_{i}-p_{j}\right\|_{2}<\frac{1}{1+\delta} \cdot W$

if the Dijkstra traversal did not reach $p_{j}$ or $d_{G}\left(p_{i}, p_{j}\right) \geq(1+\delta)\left\|p_{i}-p_{j}\right\|_{2}$ return false

return true

Notice that this algorithm only tests small neighborhoods of certain points. At first glance it seems unlikely that the spanner property can be tested by local investigations. Consider the path depicted in Figure 1. If $\delta$ is chosen appropriately, then the spanner property might be fulfilled for all pairs $\left(v_{i}, v_{j}\right)$ except for $\left(v_{1}, v_{9}\right)$. This means that the violation cannot be found by sampling only parts of the path. Surprisingly, when distinguishing spanners and graphs that are $\epsilon$-far from being a spanner, the situation is different. We show that a geometric graph cannot be $\epsilon$-far from being a spanner if it does only contain 'global' violations of the spanner property like the one in Figure 1. 


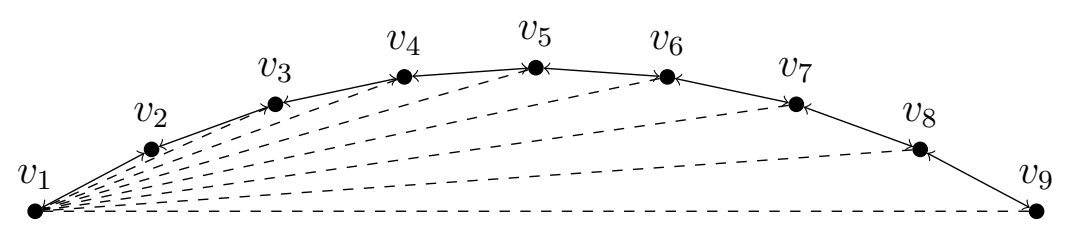

Figure 1: A curved path where the quotient of the distance in the graph and the euclidean distance grows for increasing $i$.

\section{References}

[1] N. Alon, S. Dar, M. Parnas, D. Ron. Testing of Clustering. SIAM Journal on Discrete Mathematics, 16(3): 393-417, 2003.

[2] H.-K. Ahn, M. Farshi, C. Knauer, M. Smid, Y. Wang Dilation-Optimal Edge Deletion in Polygonal Cycles. Algorithms and Computation, pp. 88-99, Springer, 2007.

[3] N. Alon, E. Fischer, I. Newman, and A. Shapira. A combinatorial characterization of the testable graph properties: it's all about regularity. SIAM Journal on Computing, 39(1): 143$167,2009$.

[4] P.K. Agarwal, R. Klein, C. Knauer, S. Langerman, P. Morin, M Sharir, M. Soss Computing the Detour and Spanning Ratio of Paths, Trees, and Cycles in 2D and 3D. Discrete and Computational Geometry, 39(1-3): 17-37, 2007.

[5] S. Arya, G. Das, M. Mount, J.S. Salowe, M. Smid. Euclidean spanners: short, thin, and lanky. Proceedings of the 27th ACM Symposium on the Theory of Computing, pp. 489-498, 1995.

[6] O. Ben-Zwi, O. Lachish, I. Newman. Lower bounds for testing Euclidean Minimum Spanning Trees. Information Processing Letters, 102(6): 219-225, 2007.

[7] T. Batu, L. Fortnow, E. Fischer, R. Kumar, R. Rubinfeld, P. White. Testing Random Variables for Independence and Identity. Proceedings of the 42nd IEEE Symposium on Foundations of Computer Science (FOCS), pp. 442-451, 2001.

[8] T. Batu, L. Fortnow, R. Rubinfeld, W. Smith, and P. White. Testing that distributions are close. Proceedings of the 41st IEEE Symposium on Foundations of Computer Science (FOCS), pp. 259-269, 2000.

[9] M. de Berg, M. van Kreveld, M. Overmars, O. Schwarzkopf. Computational Geometry Algorithms and Applications. Springer, 1997.

[10] I. Benjamini, O. Schramm, A. Shapira. Every minor-closed property of sparse graphs is testable. Proceedings of the 40th Annual ACM Symposium on Theory of Computing (STOC), pp. 393-402, 2008.

[11] E. Blais. Testing juntas nearly optimally. Proceedings of the 41st Annual ACM Symposium on Theory of Computing (STOC), pp. 151-158, 2009. 
[12] P.B. Callahan, S.R. Kosaraju. Faster algorithms for some geometric graph problems in higher dimensions. Proceedings of the 4th ACM-SIAM Symposium on Discrete Algorithms, pp. 291300, 1993.

[13] K.L. Clarkson. Approximating algorithms for shortest path motion planning. Proceedings of the 19th ACM Symposium on the Theory of Computation, pp. 56-65, 1987.

[14] A. Czumaj, A. Shapira, and C. Sohler. Testing hereditary properties of non-expanding bounded-degree graphs. SIAM Journal on Computing, 38(6): 2499-2510, 2009.

[15] A. Czumaj, C. Sohler. Property Testing with Geometric Queries. Proceedings of the 9th Annual European Symposium on Algorithms (ESA), pp. 266-277, 2001.

[16] A. Czumaj, C. Sohler, M. Ziegler. Property Testing in Computational Geometry. Proceedings of the 8th Annual European Symposium on Algorithms (ESA), pp. 155-166, 2000.

[17] A. Czumaj, C. Sohler. Testing Euclidean minimum spanning trees in the plane. ACM Transactions on Algorithms, 4(3): ,2008.

[18] F. Ergun, S. Kannan, R. Kumar, R. Rubinfeld, M. Viswanathan. Spot-Checkers. Journal of Computer and System Sciences, 60(3): 717-751, 2000.

[19] D. Eppstein, K.A. Wortman. Minimum dilation stars. Computational Geometry: Theory and Applications, 37(1): 27-37, 2007.

[20] M. Farshi, P. Giannopoulos, J. Gudmundsson Finding the best shortcut in a geometric network Proceedings of the 21th annual symposium on Computational geometry, pp. 327-335, 2005.

[21] E. Fischer, E. Lehman, I. Newman, S. Raskhodnikova, R. Rubinfeld, A. Samorodnitsky. Monotonicity testing over general poset domains. Proceedings of the 34 th Annual ACM Symposium on Theory of Computing (STOC), pp. 474-483, 2002.

[22] O. Goldreich, S. Goldwasser, E. Lehman, D. Ron, A. Samorodnitsky. Testing Monotonicity. Combinatorica, 20(3): 301-337, 2000.

[23] O. Goldreich, S. Goldwasser, D. Ron. Property Testing and its Connection to Learning and Approximation. Journal of the ACM, 45(4): 653-750, 1998.

[24] O. Goldreich, D. Ron. Property Testing in Bounded Degree Graphs. Algorithmica, 32(2): 302-343, 2008.

[25] M. Keil. Approximating the complete Euclidean graph. Lecture Notes in Computer Science, 318: 208-213, 1988.

[26] T. Kaufman, M. Sudan. Algebraic property testing: the role of invariance. Proceedings of the 40th Annual ACM Symposium on Theory of Computing (STOC), pp. 403-412, 2008.

[27] G. Narasimhan, M. Smid. Approximating the Stretch Factor of Euclidean Graphs. SIAM Journal on Computing, pp.978-989, 2000. 
[28] G. Narasimhan, M. Smid. Geometric Spanner Networks. Cambridge University Press, 2007.

[29] L. Rademacher, S. Vempala. Testing Geometric Convexity. Proceedings of the 24th Foundations of Software Technology and Theoretical Computer Science (FSTTCS), pp. 469-480, 2004.

[30] R. Rubinfeld, M. Sudan. Robust Characterizations of Polynomials with Applications to Program Testing. SIAM Journal on Computing, 25(2): 252-271, 1996. 